\title{
Negative pressure wound therapy in the treatment of surgical site infection in cardiac surgery
}

\author{
Terapia por pressão negativa no tratamento de infecção do sítio cirúrgico em cirurgia cardíaca
}

Terapia de presión negativa en el tratamiento de la infección del sitio quirúrgico en la cirugía de corazón

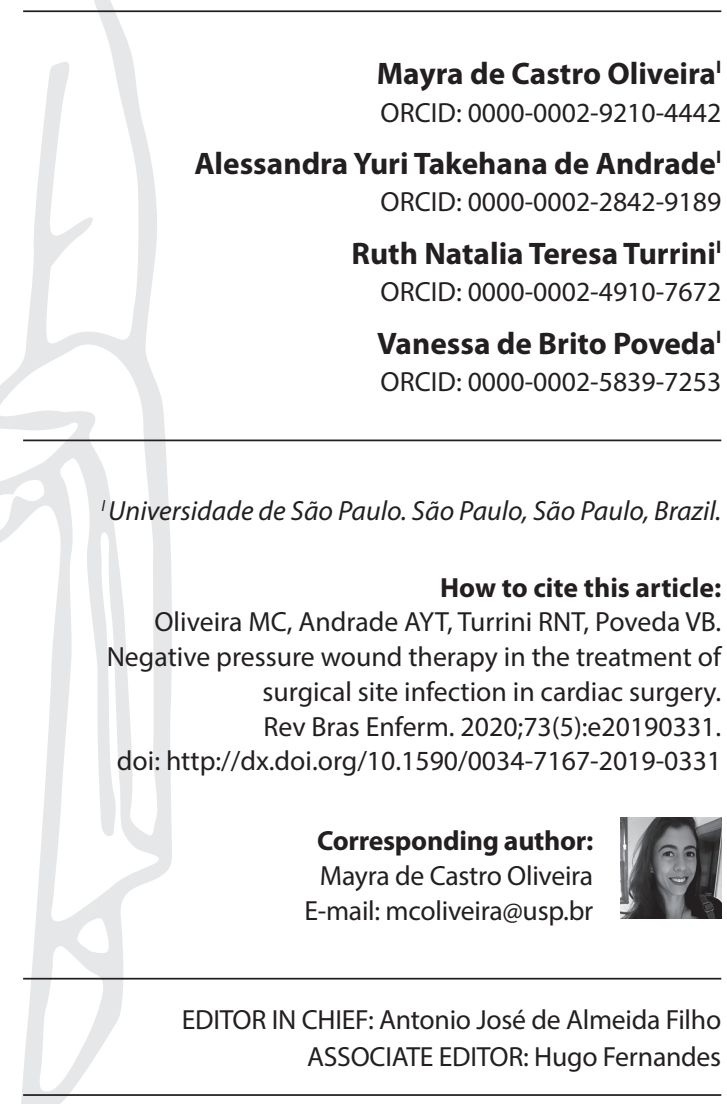

Submission: 04-19-2019

Approval: 10-12-2019

\begin{abstract}
Objectives: to describe the relationship between epidemiological and clinical characteristics of postoperative cardiac surgery patients undergoing negative pressure wound therapy for the treatment of surgical site infection. Methods: an observational, cross-sectional analytical study including a convenience sample consisting of medical records of patients undergoing sternal cardiac surgery with surgical site infection diagnosed in medical records treated by negative pressure wound therapy. Results: medical records of 117 patients, mainly submitted to myocardial revascularization surgery and with deep incisional surgical site infection $(88 ; 75.2 \%)$. Negative pressure wound therapy was used on mean for $16( \pm 9.5)$ days/patient; $1.7 \%$ had complications associated with therapy and $53.8 \%$ had discomfort, especially pain (93.6\%). The duration of therapy was related to the severity of $\mathrm{SSI}(\mathrm{p}=0.010)$ and the number of exchanges performed $(p=0.045)$. Conclusions: negative pressure wound therapy has few complications, but with discomfort to patients.

Descriptors: Surgical Wound Infection; Negative-Pressure Wound Therapy; Sternotomy; Thoracic Surgical Procedures; Cardiology.
\end{abstract}

\section{RESUMO}

Objetivos: descrever as relações entre as características epidemiológicas e clínicas de pacientes no pós-operatório de cirurgia cardíaca submetidos à terapia por pressão negativa para o tratamento de infecção do sítio cirúrgico. Métodos: estudo observacional, transversal analítico, incluindo uma amostra por conveniência composta por prontuários de pacientes submetidos à cirurgia cardíaca esternal com infecção do sítio cirúrgico diagnosticada em prontuário, tratada pela terapia por pressão negativa. Resultados: foram incluídos prontuários de 117 pacientes, submetidos principalmente à cirurgia de revascularização do miocárdio e com infecção do sítio cirúrgico incisional profunda $(88 ; 75.2 \%)$. A terapia por pressão negativa foi utilizada em média por $16( \pm 9.5)$ dias/paciente; $1.7 \%$ tiveram complicações associadas à terapia e $53.8 \%$ apresentaram desconforto, principalmente dor (93.6\%). O tempo de uso da terapia esteve relacionado à gravidade da ISC $(p=0.010)$ e à quantidade de trocas realizadas $(p=0.045)$. Conclusões: a terapia por pressão negativa apresenta poucas complicações, porém com desconforto aos pacientes. Descritores: Infecção da Ferida Cirúrgica; Tratamento de Ferimentos com Pressão Negativa; Esternotomia; Procedimentos Cirúrgicos Torácicos; Cardiologia.

\section{RESUMEN}

Objetivos: describir la relación entre las características epidemiológicas y clínicas de los pacientes con cirugía cardíaca postoperatoria sometidos a terapia de presión negativa para el tratamiento de la infección del sitio quirúrgico. Métodos: estudio analítico observacional transversal que incluye una muestra de conveniencia que consiste en registros médicos de pacientes sometidos a cirugía cardíaca esternal con infección del sitio quirúrgico diagnosticada en registros médicos tratados con terapia de presión negativa. Resultados: se incluyeron registros médicos de 117 pacientes, que se sometieron principalmente a cirugía de revascularización miocárdica e infección del sitio quirúrgico incisional profundo (88; $75.2 \%)$. La terapia de presión negativa se usó en promedio durante $16( \pm 9.5)$ días/paciente; El 1.7\% tuvo complicaciones asociadas con la terapia y el $53.8 \%$ tuvo molestias, especialmente dolor (93.6\%). La duración de la terapia se relacionó con la gravedad de SSI $(p=0.010)$ y el número de intercambios realizados $(p=0.045)$. Conclusiones: la terapia de presión negativa presenta pocas complicaciones, pero con molestias para los pacientes. Descriptores: Infección de la Herida Quirúrgica; Tratamiento de Heridas con Presión Negativa; Esternotomía; Procedimientos Quirúrgicos Torácicos; Cardiología. 


\section{INTRODUCTION}

Surgical site infection (SSI) is the most common healthcareassociated infection (HAl) among surgical patients. It accounts for $21.8 \%$ of all $\mathrm{HAl}$, associated with a $3 \%$ mortality rate, with approximately three quarters of deaths attributable directly to $\mathrm{SS} \mathrm{I}^{(1-2)}$.

The Centers for Disease Control and Prevention (CDC) has developed criteria that define and categorize SSI according to their topography, that is, the compromise by the infectious process of the different tissues opened and manipulated during the surgical procedure. They divide infections into superficial incisional SSI when it involves only skin and subcutaneous tissue; deep incisional SSI when affecting deep soft tissues such as fascia and muscle; and organ/space SSI, when it covers all space and organ manipulated during surgery ${ }^{(3)}$.

The occurrence of SSI in cardiac surgeries is related to increased morbidity and mortality, with involvement rates reaching $38.9 \%$. They increase costs, extend hospitalization, linked to the greater need for dressings, laboratory tests, antibiotic use and treatment of other complications ${ }^{(2,4-12)}$.

Negative pressure wound therapy (NPWT) is among the many therapeutic options for treating complex wounds with infection. It can be considered as an adjuvant method by local application of controlled subatmospheric pressure to accelerate the healing process by reducing edema, exudation, bacterial colonization, increased perfusion, granulation tissue formation and promotion. patient comfort by reducing daily wound handling ${ }^{(13-21)}$.

The World Health Organization (WHO) recommends only prophylactic use of NPWT to prevent SSI in previously closed, high-risk surgical incisions ${ }^{(22)}$. However, it is observed in clinical practice that the most frequent use of this technology is related to the treatment of SSI, although its use is supported by limited scientific evidence, especially for the treatment of SSI after cardiac surgery ${ }^{(23)}$. For this reason, its indication should be cautious due to the expensive device-related costs and the low quality of evidence ${ }^{(22)}$, leading to the need to explore the clinical outcomes of patients undergoing NPWT SSI treatment.

\section{OBJECTIVES}

To describe the relationship between epidemiological and clinical characteristics of postoperative cardiac surgery patients undergoing NPWT for the treatment of SSI.

\section{METHODS}

\section{Ethical aspects}

This project was approved by the Research Ethics Committee of the University of Sao Paulo Nursing School. It was prepared in accordance with the Regulatory Guidelines and Norms for Research Involving Human Beings, issued by Resolution 466 of 2012 of the National Health Council (CNS, acronym in Portuguese), safeguarding the confidentiality of information regarding the identity of participants.

\section{Design, place of study and period}

An observational cross-sectional analytical study, guided by the STROBE tool, conducted in a large hospital specialized in the clinical and surgical treatment of cardiovascular diseases in the state of Sao Paulo. Data extracted are from January 2017 to May 2018.

\section{Population or sample; inclusion and exclusion criteria}

The convenience sample consisted of 117 medical records of patients undergoing sternal cardiac surgery with SSI diagnosed in medical records. They met the diagnostic definitions proposed by the CDC and National Sanitary Surveillance Agency (ANVISA, acronym in Portuguese), dealt with by the NPWT ${ }^{(3,6)}$.

The medical records of patients over 18 years old who underwent cardiac surgery who developed SSI and were treated with NPWT were included. Medical records of patients undergoing cardiac surgery, with or without prosthesis implantation, within 30 days prior to surgery that presented SSI, and those who remained with NPWT for less than 72 hours were excluded.

\section{Study protocol}

A list of NPWT installation procedures was obtained through the Medical and Hospital Information Service of the hospital in question, which guided the search for medical records, and found 559 records. After excluding the duplicate records and applying the inclusion and exclusion criteria, 117 medical records were selected. Data collection was performed through a form prepared by the authors, containing information on clinical characteristics, health history, preoperative origin and preparation, characteristics of the anesthetic-surgical procedure, postoperative situation, surgical incision, culture and sensitivity profile, antimicrobial use and NPWT use.

Prior to the actual data collection, a pilot test was performed to adjust the proposed data collection instrument to the established objectives. Three medical records of patients who underwent cardiac surgery prior to the delimited period for this investigation were used and were therefore not included in the sample.

With regard to preoperative preparation, the routine employed by the institution consists of a bath with $2 \%$ degenerating chlorhexidine and trichotomy with electric trichotomizer (if necessary) at most two hours before surgery; antibiotic prophylaxis administered in anesthetic induction according to hospital standardization (Cefuroxime); and skin preparation with degerming $2 \%$ chlorhexidine and $0.5 \%$ alcoholic chlorhexidine before surgical incision. In addition, the sterile dressing is maintained for 24 hours after surgery.

\section{Analysis of results, and statistics}

The data obtained were tabulated in a database using Microsoft Excel software and analyzed with the help of a statistical professional using the Statistical Package for the Social Sciences (SPSS) software, 21.0.

Quantitative variables were analyzed by means and standard deviations and categorical variables by frequency distribution. The Kruskal-Wallis test was used to evaluate the relationship between SSI severity and time of therapy use and number of exchanges performed. Fisher's exact test measured the correlation between the severity of SSI and Diabetes Mellitus (DM). Pearson's test was used to verify the association between the 
time of therapy use and obesity, as well as the presence of microorganisms detected in the culture. The Wilcoxon-Mann-Whitney test was used to test whether the duration of therapy was related to readmission and discomfort. The significance level adopted was $\mathrm{p} \leq 0.05$.

\section{RESULTS}

A total of 117 patients who used NPWT as a result of sternal SSI were included. The sample consisted predominantly of women, with a mean age of 59.9 $( \pm 13.4)$ years. Regarding lifestyle habits, $6.8 \%$ of the individuals were active smokers, 29.9\% former smokers, 21.2\% were alcoholics, with a mean Body Mass Index (BMI) of $29.2( \pm 5.9) \mathrm{kg} / \mathrm{m}^{2}$. Most of the population (98.3\%) had chronic diseases, the most prevalent being: heart disease, Systemic Arterial Hypertension (SAH), DM and obesity (Table 1).

No statistical association was observed regarding obesity ( $p=0.120$ ) or presence of DM $(p=0.4504)$ and severity of SSI.

Most patients were admitted through the inpatient unit (IU), taking a mean of $6.2( \pm 8.9)$ days from admission to cardiac surgery (Table 1).

The most commonly performed surgical procedure was coronary artery bypass grafting (CABG) $(57 ; 48.7 \%)$, followed by valve surgery $(37 ; 31.6 \%)$. The use of cardiopulmonary bypass (CPB) during the surgical procedure was performed in $92.3 \%$ of the situations; with a mean of $101.2( \pm 52.1)$ minutes of CPB $(p=0.740)$ and $78.2( \pm 36.7)$ minutes of anoxia $(p=0.868)$, with no statistical significance regarding the type of SSI. The hospital readmission rate for SSI was $48.7 \%$ and death equal to $20.5 \%$ (Table 1). It was not possible to infer statistical association between length of stay $(p=0.383)$ and readmission time $(p=0.201)$ with SSI classification.

Regarding the classification of SSI, the deep incisional type prevailed $(88 ; 75.2 \%)$, followed by organ/space $(27 ; 23.1 \%)$ and superficial incisional SSI $(2 ; 1.7 \%)$. The characteristics presented by the patients that determine each type of SSI are shown in Table 2.

NPWT was used on mean for 16 ( \pm 9.5$)$ days in each patient, with minimum use time equal to five days and maximum time of 64 days, and on mean $2.2( \pm 0.8)$ exchanges. Exudate drained during therapy has often been described as serohematic (58.4\%). More than half $(53.8 \%)$ of the individuals had discomfort related to therapy, the main one being pain (93.6\%). Only $1.7 \%$ had complications resulting from the use of therapy, which are considered as occurrences requiring immediate invasive intervention by the health team, such as hemorrhage (Table 3 ).
Table 1 - Clinical and surgical characteristics of patients undergoing cardiac surgery who developed SSI, São Paulo, São Paulo, Brazil, 2017-2018

\begin{tabular}{|c|c|}
\hline Variables & $n=117$ \\
\hline \multicolumn{2}{|l|}{ Clinical characteristics } \\
\hline Age, years, mean $( \pm S D)$ & $59.9( \pm 13.4)$ \\
\hline Weight, kg, mean ( \pm SD) & $78.4( \pm 17.0)$ \\
\hline $\mathrm{BMI}, \mathrm{kg} / \mathrm{m} 2$, mean $( \pm \mathrm{SD})$ & $29.2( \pm 5.9)$ \\
\hline Female gender, n (\%) & $60(51.3)$ \\
\hline Male gender, $\mathrm{n}(\%)$ & $57(48.7)$ \\
\hline Former smoker, n (\%) & 35 (29.9) \\
\hline Smoker, n (\%) & $8(6.8)$ \\
\hline Ethylism, n (\%) & $7(21.2)$ \\
\hline \multicolumn{2}{|l|}{ Chronic diseases } \\
\hline Heart disease, $n(\%)$ & $109(94.8)$ \\
\hline $\mathrm{SAH}, \mathrm{n}(\%)$ & $84(73.0)$ \\
\hline DM, n (\%) & $53(46.1)$ \\
\hline Obesity, n (\%) & $52(44.8)$ \\
\hline Hypothyroidism, n (\%) & $11(9.6)$ \\
\hline Non-dialytic CKD, n (\%) & $10(8.7)$ \\
\hline Dialytic CKD, n (\%) & $2(1.7)$ \\
\hline Asthma, $\mathrm{n}(\%)$ & $1(0.9)$ \\
\hline \multicolumn{2}{|l|}{ Origin and preoperative preparation } \\
\hline Inpatient Unit, n (\%) & $81(69.2)$ \\
\hline ICU, n (\%) & $29(24.8)$ \\
\hline ER, n (\%) & $7(6.0)$ \\
\hline Previous corticosteroid use, $\mathrm{n}(\%)$ & $6(5.1)$ \\
\hline \multicolumn{2}{|l|}{ Characteristics of anesthetic-surgical procedure } \\
\hline CABG, n (\%) & $57(48.7)$ \\
\hline Valve surgery, n (\%) & 37 (31.6) \\
\hline CABG + valve, $\mathrm{n}(\%)$ & $8(6.8)$ \\
\hline Heart transplantation, n (\%) & $5(4.3)$ \\
\hline Aortic surgery, n (\%) & $4(3.4)$ \\
\hline Aortic surgery + valve, n (\%) & $1(0.9)$ \\
\hline Other, n (\%) & $5(4.3)$ \\
\hline CPB use, $\mathrm{n}(\%)$ & $108(92.3)$ \\
\hline CPB time, minutes, mean ( \pm SD) & $101.2( \pm 52.1)$ \\
\hline Anoxia time, minutes, mean $( \pm S D)$ & $78.2( \pm 36.7)$ \\
\hline ICU stay, days, mean ( \pm SD) & $14.9( \pm 38.7)$ \\
\hline Total length of stay, days, mean ( \pm SD) & $41.4( \pm 35.4)$ \\
\hline Time between surgery and dressing installation, days, mean $( \pm$ SD) & $35.5( \pm 40.2)$ \\
\hline ICU readmission, $\mathrm{n}(\%)$ & $26(22.2)$ \\
\hline Reoperation, n (\%) & $15(12.8)$ \\
\hline Occurrence of readmission by SSI, $\mathrm{n}(\%)$ & $57(48.7)$ \\
\hline Readmission time, days, mean ( \pm SD) & $43.6( \pm 21.8)$ \\
\hline Deaths, n (\%) & $24(20.5)$ \\
\hline
\end{tabular}

Note: SSI - Surgical Site Infection; SD - Standard Deviation; BMI - Body Mass Index; SAH - Systemic Arterial Hypertension; DM - Diabetes Mellitus; CKD - Chronic Kidney Disease; ICU - Intensive Care Unit; ER - Emergency Room; CABG - Coronary Artery Bypass Grafting; CPB - Cardiopulmonary Bypass.

Table 2 - Distribution of SSI according to diagnostic criteria, materials collected for culture and microorganisms isolated from SSI patients after cardiac surgery, São Paulo, São Paulo, Brazil, 2017-2018

\begin{tabular}{lc}
\hline Variables & $\mathbf{n = 1 1 7}$ \\
\hline Deep incisional SSI, n (\%) & $88(75.2)$ \\
Organ/space SSI, n (\%) & $27(23.1)$ \\
Superficial incisional SSI, n (\%) & $2(1.7)$ \\
Diagnostic criteria & \\
Positive MO culture, n (\%) & $106(90.6)$ \\
Purulent drainage, n (\%) & $80(68.4)$ \\
Dehiscence, n (\%) & $56(47.9)$ \\
Pain, $\mathrm{n}(\%)$ & $35(29.9)$ \\
Fever, n (\%) & $11(9.4)$ \\
Abscess, $\mathrm{n}(\%)$ & $9(7.7)$ \\
Edema, $\mathrm{n}(\%)$ & - \\
Hyperemia, n (\%) & $3(2.6)$ \\
Heat, $\mathrm{n}(\%)$ & $2(1.7)$ \\
\hline
\end{tabular}


Table 2 (concluded)

\begin{tabular}{lc}
\hline Variables & $\mathbf{n = 1 1 7}$ \\
\hline Material collected & \\
SW secretion, $\mathrm{n}(\%)$ & $98(83.8)$ \\
SW fragment, $\mathrm{n}(\%)$ & $74(63.3)$ \\
Pericardial fluid, $\mathrm{n}(\%)$ & $2(1.7)$ \\
Presence of isolated MO in culture, $\mathrm{n}(\%)$ & $106(90.6)$ \\
Staphylococcus, $\mathrm{n}(\%)$ & $70(59.8)$ \\
Klebsiella, $\mathrm{n}(\%)$ & $25(21.4)$ \\
Candida, $\mathrm{n}(\%)$ & $23(19.7)$ \\
Enterococcus, $\mathrm{n}(\%)$ & $13(11.1)$ \\
Pseudomonas, $\mathrm{n}(\%)$ & $10(8.5)$ \\
Streptococcus, $\mathrm{n}(\%)$ & $8(6.8)$ \\
Serratia, $\mathrm{n}(\%)$ & $7(6.0)$ \\
Cultibacterium, $\mathrm{n}(\%)$ & $7(6.0)$ \\
Enterobacter, $\mathrm{n}(\%)$ & $7(6.0)$ \\
Escherichia, $\mathrm{n}(\%)$ & $6(5.1)$ \\
Citrobacter, $\mathrm{n}(\%)$ & $4(3.4)$ \\
Stenotrophomonas, n (\%) & $3(2.6)$ \\
Proteus, $\mathrm{n}(\%)$ & $3(2.6)$ \\
Bacillus, $\mathrm{n}(\%)$ & $2(1.7)$ \\
Acinetobacter, $\mathrm{n}(\%)$ & $2(1.7)$ \\
Aspergillus, $\mathrm{n}(\%)$ & $2(1.7)$ \\
Others* & $8(6.8)$ \\
\hline
\end{tabular}

Note: SSI-Surgical Site Infection; MO - microorganism; SW-Surgical Wound; *Included in other category: Actinomyces, Finegoldia, Corynebacterium, Eubacterium, Bacteroides, Veillonella, Prevotella, Rhodotorula.

Table 3 - Characteristics of the use of NPWT in SSI patients after cardiac surgery, São Paulo, São Paulo, Brazil, 2017-2018

\begin{tabular}{lc}
\hline Variables & $\mathbf{n = 1 1 7}$ \\
\hline Therapy use time, days, mean $( \pm \mathrm{SD})$ & $16( \pm 9.5)$ \\
Number of exchanges, mean $( \pm \mathrm{SD})$ & $2.2( \pm 0.8)$ \\
Characteristic of drained exudate & \\
Serohematic, $\mathrm{n}(\%)$ & $59(58.4)$ \\
Hematic, $\mathrm{n}(\%)$ & $34(33.7)$ \\
Serous, $\mathrm{n}(\%)$ & $4(4.0)$ \\
Purulent, $\mathrm{n}(\%)$ & $3(3.0)$ \\
Piohematic, $\mathrm{n}(\%)$ & $1(1.0)$ \\
Presence of therapy-related discomfort, $\mathrm{n}(\%)$ & $63(53.8)$ \\
Pain, $\mathrm{n}(\%)$ & $59(93.6)$ \\
Bleeding, $\mathrm{n}(\%)$ & $3(4.8)$ \\
Mobility restriction, $\mathrm{n}(\%)$ & $1(1.6)$ \\
Itching, $\mathrm{n}(\%)$ & $1(1.6)$ \\
Presence of therapy-related complications, $\mathrm{n}(\%)$ & $2(1.7)$ \\
\hline
\end{tabular}

Note: NPWT - Negative Pressure Wound Therapy; SSI: Surgical Site Infection; SD: Standard Deviation.
The duration of use of NPWT was related to the severity of $\mathrm{SSI}$, that is, the more severe the longer the therapy $(p=0.010)$, as well as the number of system changes performed $(p=0.045)$.

In all cases, culture and antibiogram were performed, using as secretion material and surgical wound (SW) fragment and pericardial fluid. Of these, $90.6 \%$ had microorganisms (MOs) detected in the sample, and the most commonly found genera were Staphylococcus (59.8\%), Klebsiella (21.4\%), and Candida (19.7\%) (Table 2). Those who had no positive culture were diagnosed with SSI through clinical signs. Staphylococcus $(p=0.050)$, Klebsiella $(p=0.040)$, Stenotrophomonas $(p=<0.001)$, Proteus $(p=0.011)$, and Enterobacter $(p=0.013)$ were found to be associated with longer NPWT. In addition, the presence of Aspergillus $(p=<0.001)$ was associated with longer hospital stays. The sensitivity and resistance profile of the isolated MOs is shown in Table 4.

The glycopeptide, carbapenems and aminopenicillin drugs were the most commonly used postoperatively (Table 5).

\section{DISCUSSION}

The results of the present study demonstrated that the time of use of NPWT and the amount of dressing changes is directly related to the severity of SSI. The therapy was used on mean for $16( \pm 9.5)$ days/patient and changed for $2.2( \pm 0.8)$ times. In addition, there was a statistical association between longer NPWT use and the occurrence of Staphylococcus, Klebsiella, Stenotrophomonas, Proteus, and Enterobacter-related SSIs, with a mean ICU stay of 14.9 days and a mean total hospital stay of 41.4 days.

Table 4 - Sensitivity and resistance profile of microorganisms isolated by antimicrobial groups among SSI patients after cardiac surgery, São Paulo, São Paulo, Brazil, 2017-2018

\begin{tabular}{|c|c|c|c|c|c|c|c|c|c|c|c|c|c|}
\hline Groups & $\frac{\text { 뜬 }}{\frac{0}{0}}$ & 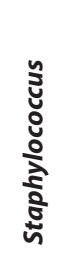 & 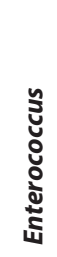 & 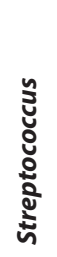 & 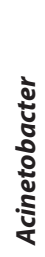 & 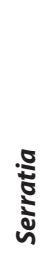 & 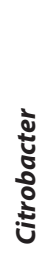 & 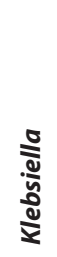 & 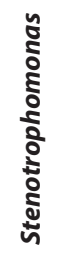 & 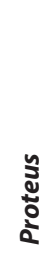 & 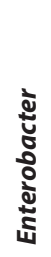 & 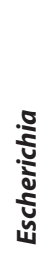 & 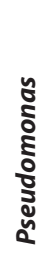 \\
\hline Aminopenicillins & $\begin{array}{l}\mathrm{S} \\
\mathrm{R}\end{array}$ & $\begin{array}{l}32 \\
67\end{array}$ & $\begin{array}{l}6 \\
7\end{array}$ & $\begin{array}{l}4 \\
-\end{array}$ & $\begin{array}{l}1 \\
1\end{array}$ & $\begin{array}{l}3 \\
7\end{array}$ & $\begin{array}{l}4 \\
4\end{array}$ & $\begin{array}{c}8 \\
24\end{array}$ & - & $\begin{array}{l}3 \\
2\end{array}$ & $\begin{array}{l}2 \\
6\end{array}$ & $\begin{array}{l}5 \\
4\end{array}$ & $\begin{array}{l}6 \\
-\end{array}$ \\
\hline Cephalosporins & $\begin{array}{l}\mathrm{S} \\
\mathrm{R}\end{array}$ & - & $\begin{array}{l}- \\
-\end{array}$ & $\begin{array}{l}6 \\
-\end{array}$ & $\begin{array}{l}1 \\
1\end{array}$ & $\begin{array}{l}4 \\
7\end{array}$ & $\begin{array}{l}4 \\
2\end{array}$ & $\begin{array}{c}9 \\
20\end{array}$ & $\begin{array}{l}- \\
-\end{array}$ & $\begin{array}{l}3 \\
1\end{array}$ & $\begin{array}{l}4 \\
6\end{array}$ & $\begin{array}{l}5 \\
2\end{array}$ & $\begin{array}{l}7 \\
1\end{array}$ \\
\hline Carbapenems & $\begin{array}{l}\mathrm{S} \\
\mathrm{R}\end{array}$ & - & - & - & $\begin{array}{l}1 \\
1\end{array}$ & $\begin{array}{l}4 \\
3\end{array}$ & $\begin{array}{l}4 \\
-\end{array}$ & $\begin{array}{l}10 \\
15\end{array}$ & - & $\begin{array}{l}3 \\
-\end{array}$ & $\begin{array}{l}7 \\
-\end{array}$ & $\begin{array}{l}5 \\
1\end{array}$ & $\begin{array}{l}3 \\
5\end{array}$ \\
\hline Aminoglycosides & $\begin{array}{l}\mathrm{S} \\
\mathrm{R}\end{array}$ & $\begin{array}{c}58 \\
9\end{array}$ & $\begin{array}{c}10 \\
4\end{array}$ & - & $\begin{array}{l}2 \\
1\end{array}$ & $\begin{array}{l}4 \\
4\end{array}$ & $\begin{array}{l}4 \\
-\end{array}$ & $\begin{array}{l}23 \\
11\end{array}$ & - & $\begin{array}{l}3 \\
-\end{array}$ & $\begin{array}{l}7 \\
3\end{array}$ & $\begin{array}{l}6 \\
1\end{array}$ & $\begin{array}{l}7 \\
1\end{array}$ \\
\hline Chloramphenicol & $\begin{array}{l}\mathrm{S} \\
\mathrm{R}\end{array}$ & - & - & $\begin{array}{l}6 \\
-\end{array}$ & - & - & - & - & - & - & - & - & - \\
\hline
\end{tabular}




\begin{tabular}{|c|c|c|c|c|c|c|c|c|c|c|c|c|c|}
\hline Groups & 을 & 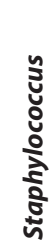 & 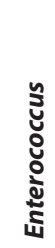 & 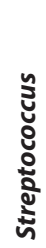 & 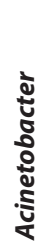 & 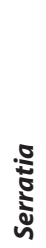 & 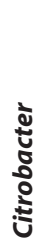 & 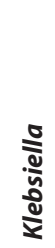 & 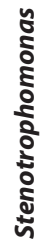 & 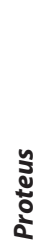 & 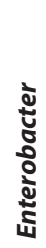 & 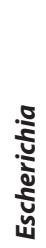 & 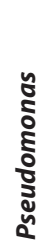 \\
\hline \multirow[t]{2}{*}{ Macrolids } & $S$ & 64 & - & 2 & - & - & - & - & - & - & - & - & - \\
\hline & $\mathrm{R}$ & 49 & - & 2 & - & - & - & - & - & - & - & - & - \\
\hline \multirow[t]{2}{*}{ Quinolones } & $\mathrm{S}$ & 31 & 1 & 2 & 1 & 4 & 4 & 8 & 2 & 3 & 4 & 2 & 6 \\
\hline & $\mathrm{R}$ & 43 & - & - & 1 & 3 & - & 16 & 1 & - & 2 & 4 & 2 \\
\hline \multirow[t]{2}{*}{ Glycopeptides } & $\mathrm{S}$ & 68 & 6 & 6 & - & - & - & - & - & - & - & - & - \\
\hline & $\mathrm{R}$ & 7 & 7 & - & - & - & - & - & - & - & - & - & - \\
\hline \multirow[t]{2}{*}{ Oxazolidinones } & $\mathrm{S}$ & 68 & 12 & 4 & - & - & - & - & - & - & - & - & - \\
\hline & $\mathrm{R}$ & - & - & - & - & - & - & - & - & - & - & - & - \\
\hline \multirow[t]{2}{*}{ Polymyxins } & $\mathrm{S}$ & - & - & - & 1 & - & 4 & 15 & - & - & 3 & 3 & 8 \\
\hline & $\mathrm{R}$ & - & - & - & - & - & - & 5 & - & - & - & - & - \\
\hline \multirow[t]{2}{*}{ Sulfonamides } & $\mathrm{S}$ & 40 & - & - & - & - & - & - & 3 & - & - & - & - \\
\hline & $\mathrm{R}$ & 34 & - & - & - & - & - & - & - & - & - & - & - \\
\hline \multirow[t]{2}{*}{ Lincosamines } & $\mathrm{S}$ & 27 & - & 6 & - & - & - & - & - & - & - & - & - \\
\hline & $\mathrm{R}$ & 43 & - & - & - & - & - & - & - & - & - & - & - \\
\hline \multirow[t]{2}{*}{ Glycylcycline } & $S$ & 64 & 12 & - & 1 & - & 3 & 16 & - & - & 6 & 6 & - \\
\hline & $\mathrm{R}$ & - & - & - & 1 & 2 & - & 9 & - & - & 1 & - & - \\
\hline
\end{tabular}

Note: SSI: Surgical Site Infection; S: Sensitive; R: Resistant.

Table 5 - Postoperative frequency of antimicrobial use of SSI patients after cardiac surgery. Sao Paulo, Sao Paulo, Brazil, 2017-2018

\begin{tabular}{lc}
\hline Groups & $\mathbf{n = 1 1 7}$ \\
\hline Glycopeptides, $\mathrm{n}(\%)$ & $106(90.60)$ \\
Carbapenems, $\mathrm{n}(\%)$ & $75(64.10)$ \\
Aminopenicillins, $\mathrm{n}(\%)$ & $62(52.99)$ \\
Quinolones, $\mathrm{n}(\%)$ & $57(48.72)$ \\
Triazolic antifungals, $\mathrm{n}(\%)$ & $36(30.77)$ \\
Aminoglycosides, $\mathrm{n}(\%)$ & $29(24.79)$ \\
Polymyxins, $\mathrm{n}(\%)$ & $23(19.66)$ \\
Cephalosporins, $\mathrm{n}(\%)$ & $20(17.09)$ \\
Oxazolidinones, $\mathrm{n}(\%)$ & $20(17.09)$ \\
Lipopeptides, $\mathrm{n}(\%)$ & $20(17.09)$ \\
Nitroimidazoles, $\mathrm{n}(\%)$ & $13(11.11)$ \\
Glycylcycline, $\mathrm{n}(\%)$ & $10(8.55)$ \\
Echinocandins, $\mathrm{n}(\%)$ & $8(6.84)$ \\
Sulfonamides, $\mathrm{n}(\%)$ & $7(5.98)$ \\
Macrolides, $\mathrm{n}(\%)$ & $6(5.13)$ \\
Lincosamines, $\mathrm{n}(\%)$ & $2(1.71)$ \\
Chloramphenicol, $\mathrm{n}(\%)$ & $1(0.85)$ \\
Polyenic antifungals, $\mathrm{n}(\%)$ & $1(0.85)$ \\
\hline Note: SSl: Surgical Site Infection. & \\
\hline
\end{tabular}

Interestingly, this result is close to those found in previous studies in which the mean duration of therapy was longer for cases of deep incisional SSI, with treatment means ranging from 13 to 49.4 days $^{(13,17,24)}$.

Unlike in this study, in which NPWT was reserved for more complicated infectious conditions, the National Institute of Cardiovascular Diseases in Slovakia has mostly used NPWT to treat less complex SSI, especially superficial incisional SSI, in $89 \%$ of the evaluated cases, followed by $10 \%$ deep and $1 \%$ organ/space ${ }^{(25)}$.

It is noteworthy that a recent integrative review analyzing eight retrospective observational studies comparing the use of NPWT with conventional dressings to treat mediastinitis concluded that NPWT was superior to other technologies. NPWT contributed to increased healing speed and low rates of reinfection, mortality or hospital readmission ${ }^{(23)}$.

Considering the multifactorial aspects involved in SSI, in general, the presence of associated comorbidities is an aggravating factor in the development and recovery of SSI. These include hypertension, diabetes mellitus, obesity and smoking, which in addition to systemically influencing metabolism, are still unfavorable for healing ${ }^{(25-26)}$. Thus, when compared to patients who did not use therapy, the literature showed that the mean BMI and DM prevalence is significantly higher in those who used $\mathrm{NPWT}^{(13)}$.

National research conducted in Minas Gerais also found that the microorganisms most related to infection were Staphylococcus and Klebsiella, an aspect observed in other countries such as Slovakia and Japan ${ }^{(4,17,25,27)}$. In Poland, the most frequent infections were caused by Gram-negative rods, and the most used antimicrobial therapy was the cephalosporin class, followed by glycopeptides and carbapenems ${ }^{(13)}$.

Analyzing patients undergoing sternal surgeries, with SSI and not submitted to NPWT, it is observed that, on mean, these patients remained in the ICU 19.1 days and were hospitalized for periods between 33 and 67 days. NPWT is suggested to reduce ICU stay compared to conventional treatment ${ }^{(4,11)}$. Studies comparing the use of NPWT to another type of intervention found a significantly shorter ICU stay ${ }^{(28-29)}$ and a tendency to shorter length of stay in those treated by NPWT ${ }^{(28)}$.

The importance of post-discharge surveillance is emphasized, since the long interval between the date of surgery and the diagnosis of infection suggests that the patient is discharged before the onset of the first infectious signs and symptoms. This justifies the high readmission rates in the present study $(48.7 \%)$, higher than those found in previous studies $(23.8 \%)^{(26)}$. This is possibly also related to the fact that in the current investigation, the time elapsed between surgery and the onset of NPWT was approximately 35.5 days, a result higher than that found in the literature (mean 22 and 29.3 days) $)^{(13,17)}$. 
A multicenter study comparing health care costs after cardiac surgery found that SSI patients cost healthcare services five times the amount spent among patients without infection ${ }^{(11)}$. Similarly, treatment costs for patients using NPWT are significantly higher than those who did not use NPWT ${ }^{(13)}$.

The prevalence of complications related to NPWT treatment, such as bleeding, was estimated at $1.7 \%$ in this study, which may be linked to the fact that the most prevalent therapy-related discomfort was pain, followed by bleeding. However, it is interesting to note that complications such as those found in the present study were considered absent in previous investigations ${ }^{(13,17)}$. Others are suggested, such as damage to adjacent blood vessels, granulation tissue hypertrophy and long-term use, increased anxiety, decreased quality of life, and malnutrition ${ }^{(30-31)}$.

A study analyzing 73 medical records of patients who developed deep incisional SSI after cardiovascular surgery showed a mortality rate of up to $33 \%$ for patients using NPWT ${ }^{(27)}$. Such data may be associated with the severity of the clinical condition due to infection and, consequently, higher risk of death. However, this same study demonstrated that NPWT was associated with a significant reduction in in-hospital mortality caused by deep incisional SSI when compared to patients who did not use the therapy ${ }^{(27)}$.

Scientific literature indicates antimicrobial-associated NPWT as a first instance treatment for sternal SSIs that do not present with sternal instability ${ }^{(17,32)}$. There are also those that indicate its use in closed wounds in order to optimize treatment and healing, and prevent infections in those patients who already have risk factors for surgical incision complications ${ }^{(33)}$. In addition, early treatment and prevention strategies for SSI have been shown to reduce mortality ${ }^{(32)}$.

Finally, it is emphasized that much remains to be investigated to accurately establish criteria for therapy indication, potential complications, and especially to determine the quality of benefits associated with the use of NPWT in infected complex surgical wounds, such as those from cardiac procedures compared to treatments already employed.

\section{Study limitations}

Limitations include the analysis of a convenience sample, limited to a single specialized treatment center. It is believed that the replication of this study, comparing patient profiles across institutions, may provide further insights into the discussion of the therapeutic use of NPWT in complex wounds.

\section{Contributions to nursing, health or public policies}

The recognition of the characteristics related to the use of NPWT in this category of patients, especially the mean exchange, microorganisms of greater epidemiological relevance and complications may help nurses to make decisions regarding nursing care to patients during the treatment of SSIs.

\section{CONCLUSIONS}

NPWT was used for about $16( \pm 9.5)$ days/patient, with a mean of $2.2( \pm 0.8)$ exchanges and was mainly applied to the treatment of deep incisional and organ/space SSI. Only $1.7 \%$ had complications resulting from the use of therapy, although $53.8 \%$ had discomfort, especially pain.

The duration of use of NPWT was related to the severity of SSI and the number of changes made. Staphylococcus, Klebsiella, Stenotrophomonas, Proteus, and Enterobacter were found to be associated with longer NPWT.

Therefore, NPWT has been employed in cases of more severe infections, with few complications associated with therapy, but with discomfort for patients.

\section{REFERENCES}

1. Magill SS, Edwards JR, Bamberg W, Beldavs ZG, Dumyati G, Kainer MA et al. Multistate point-prevalence survey of health care-associated infections. N Engl J Med. 2014;370(13):1198-208. doi: 10.1056/NEJMoa1306801

2. Awad SS. Adherence to surgical care improvement project measures and postoperative surgical site infections. Surg Infect (Larchmt). 2012;13(4): 234-7. doi: 10.1089/sur.2012.131

3. Center for Disease Control and Prevention Surgical Site Infection (SSI) Event. 2017 [cited 2017 Sep 7]. Available from: https://www.cdc.gov/ nhsn/pdfs/pscmanual/9pscssicurrent.pdf

4. Silva QCG, Barbosa MH. Risk factors for surgical site infection in cardiac surgery. Acta Paul Enferm. 2012;25(spe2):89-95. doi: 10.1590/ S0103-21002012000900014

5. Schweizer ML, Cullen JJ, Perencevich EN, Vaughan Sarrazin MS. Costs associated with surgical site infections in Veterans Affairs Hospital. JAMA Surg. 2014;149(6):575-81. doi: 10.1001/jamasurg.2013.4663

6. Agência Nacional de Vigilância Sanitária. Critérios diagnósticos de infecção relacionada a assistência à saúde. 2 ed. Brasília: ANVISA, 2017 [cited 2017 Sep7]. Available from: http://portal.anvisa.gov.br/documents/33852/271855/Crit\%C3\%A9rios+Diagn\%C3\%B3sticos+de+IRAS+ $+2+$ Ed/b9cd1e23-427b-496f-b91a-bbdae23ece63

7. Harrop JS, Styliaras JC, Ooi YC, Radcliff KE, Vaccaro AR, Wu C. Contributing factors to surgical site infections. J Am Acad Orthop Surg. 2012;20(2):94-101. doi: 10.5435/JAAOS-20-02-094

8. Kusachi S, Kashimura N, Konishi T, Shimizu J, Kusunoki M, Oka M et al. Length of stay and cost for surgical site infection after abdominal and cardiac surgery in Japanese hospitals: multi-center surveillance. Surg Infect (Larchmt). 2012;13:257-65. doi: 10.1089/sur.2011.007

9. Shepard J, Ward W, Milstone A, Carlson T, Frederick J, Hadhazy E et al. Financial impact of surgical site infections on hospitals: the hospital management perspective. JAMA Surg. 2013;148(10):907-914. doi: 10.1001/jamasurg.2013.2246 
10. Kripalani S, Theobald CN, Anctil B, Vasilevskis EE. Reducing hospital readmission rates: current strategies and future directions. Annu Rev Med. 2014;65:471-85. doi: 10.1146/annurev-med-022613-090415

11. Kobayashi J, Kusachi S, Sawa Y, Motomura N, Imoto Y, Makuuchi H et al. Socioeconomic effects of surgical site infection after cardiac surgery in Japan. Surg Today. 2015 [cited 2017 Sep 7];45(4):422-8. doi: 10.1007/s00595-014-0969-2

12. Fusco SFB, Massarico NM, Alves MVMFF, Fortaleza CMCB, Pavan ECP, Palhares VC et al. Surgical site infection and its risk factors in colon surgeries. Rev Esc Enferm USP. 2016;50(1):43-9. doi: http://dx.doi.org/10.1590/S0080-623420160000100006

13. Listewnik MJ, Sielicki P, Mokrzycki K, Biskupski A, Brykczyński M., The use of Vacuum-Assisted Closure in purulent complications and difficult-to-heal wounds in cardiac surgery. Adv Clin Exp Med. 2015;24(4):643-50. doi: 10.17219/acem/28111

14. Anghel EL, Kim PJ. Negative-pressure wound therapy: a comprehensive review of the evidence. Plast Reconstr Surg. 2016;138(3 Suppl):129S37S. doi: $10.1097 /$ PRS. 0000000000002645

15. Camargo PAB, Bertanha M, Moura R, Jaldin RG, Yoshida RA, Pimenta REF et al. Using vacuum therapy as an adjunctive treatment for healing of infected surgical sites. J Vasc Bras. 2016;15(4):312-6. doi: http://dx.doi.org/10.1590/1677-5449.002816

16. Lima RVKS, Coltro PS, Farina Júnior JA. Terapia por pressão negativa no tratamento de feridas complexas. Rev Col Bras Cir. 2017;44(1):81-93. doi: 10.1590/0100-69912017001001

17. Yoshimoto A, Inoue T, Fujisaki M, Morizumi S, Suematsu Y. Efficacy of vacuum-assisted closure therapy on rehabilitation during the treatment for surgical site infection after cardiovascular surgery. Gen Thorac Cardiovasc Surg. 2016;64(8):464-9. doi: 10.1007/s11748-016-0664-x

18. Bruhin A, Ferreira F, Chariker M, Smith J, Runkel N. Systematic review and evidence based recommendations for the use of negative pressure wound therapy in the open abdomen. Int J Surg. 2014;12(10):1105-14. doi: 10.1016/j.ijsu.2014.08.396

19. Guffanti A. Negative pressure wound therapy in the treatment of diabetic foot ulcers: a systematic review of the literature. J Wound Ostomy Continence Nurs. 2014;41(3):233-7. doi: 10.1097/WON.0000000000000021

20. Monsen C, Wann-Hansson C, Wictorsson C, Acosta S. Vacuum-assisted wound closure versus alginate for the treatment of deep perivascular wound infections in the groin after vascular surgery. J Vasc Surg. 2014;59(1):145-51. doi: 10.1016/j.jvs.2013.06.073

21. Jones DA, Neves Filho WV, Guimarães JS, Castro DA, Ferracini AM. The use of negative pressure wound therapy in the treatment of infected wounds. Case studies. Rev Bras Ortop. 2016;51(6):646-651. doi: http://dx.doi.org/10.1016/j.rboe.2016.10.014

22. WHO. Global guidelines for the prevention of surgical site infection. World Health Organization, 2016 [cited 2017 Sep 7]. Available from: http://www.who.int/gpsc/global-guidelines-web.pdf

23. Frantz CV, Stocco JGD, Ribeiro ACG, Vieira ALG. Curativos indicados no tratamento de mediastinite após cirurgia cardíaca: revisão integrativa. Texto Contexto Enferm. 2019;28:e20180073. doi: 10.1590/1980-265x-tce-2018-0073

24. Chen Y, Almeida AA, Mitnovetski S, Goldstein J, Lowe C, Smith JA. Managing deep sternal wound infections with vacuum-assisted closure. ANZ J Surg. 2008;78(5):333-6. doi: 10.1111/j.1445-2197.2008.04467.x

25. Hulman M, Bezak B, Artemiou P, Cikrai R. Wound infections after median sternotomy treated by VAC therapy, summary of results, and risk factor analysis. Bratisl Med J. 2017;118(12):736-9. doi: 10.4149/BLL_2017_139

26. Magalhães MGPA, Alves LMO, Alcantara LFMA, Bezerra SMMS. Mediastinite pós-cirúrgica em um Hospital Cardiológico de Recife: contribuições para a assistência de enfermagem. Rev Esc Enferm USP. 2012;46(4):865-71. doi: http://dx.doi.org/10.1590/S0080-62342012000400012

27. Morisaki A, Hosono M, Murakami T, Sakaguchi M, Suehiro Y, Nishimura S et al. Effect of negative pressure wound therapy followed by tissue flaps for deep sternal wound infection after cardiovascular surgery: propensity score matching analysis. Interact Cardiovasc Thorac Surg. 2016;23(3):397-402. doi: 10.1093/icvts/ivw141

28. Nickl S, Steindl J, Langthaler D, Nierlich-Hold A, Pona I, Hitzl W et al. First experiences with incisional negative pressure wound therapy in a high-risk poststernotomy patient population treated with pectoralis major muscle flap for deep sternal wound infection. J Reconstr Microsurg. 2018;34(1):1-7. doi: 10.1055/s-0037-1605379

29. Lo Torto F, Monfrecola A, Kaciulyte J, Ciudad P, Casella D, Ribuffo D, et al. Preliminary result with incisional negative pressure wound therapy and pectoralis major muscle flap for median sternotomy wound infection in a high-risk patient population. Int Wound J. $2017 ; 14(6): 1335-9$. doi: 10.1111/iwj.12808

30. Malmsjö M, Gustafsson L, Lindstedt S, Gesslein B, Ingemansson R. The effects of variable, intermittent, and continuous negative pressure wound therapy, using foam or gauze, on wound contraction, granulation tissue formation, and ingrowth into the wound filler. Eplasty [Internet]. 2012 [cited 2017 Oct 15];11:42-54. Available from: https://www.ncbi.nlm.nih.gov/pubmed/22292101?dopt=Abstract

31. Li Z, Yu A. Complications of negative pressure wound therapy: a mini review. Wound Repair Regen. 2014;22(4):457-61. doi: 10.1111/wrr.12190

32. Kaul P. Sternal reconstruction after post-sternotomy mediastinitis. J Cardiothorac Surg. 2017;12:94. doi: 10.1186/s13019-017-0656-7

33. Witt-Majchrzak A, Żelazny P, Snarska J. Preliminary outcome of treatment of postoperative primarily closed sternotomy wounds treated using negative pressure wound therapy. Pol Przegl Chir. 2014;86(10):456-65. doi: 10.2478/pjs-2014-0082 